\title{
Complete genome sequences of two Enterococcus faecium strains and comparative genomic analysis
}

\author{
YONG-QI GAN, TAO ZHANG, YONG-QIANG GAN, ZHUANG ZHAO and BIN ZHU \\ Guangxi Institute for Food and Drug Control, Nanning, Guangxi Zhuang Autonomous Region 530021, P.R. China
}

Received December 1, 2018; Accepted August 12, 2019

DOI: $10.3892 /$ etm.2020.8447

\begin{abstract}
Enterococci are used for improvement of the intestinal environment and have clinical benefits. Enterococcus faecalis and Enterococcus faecium have similar morphologies, leading to confusion between the two species. In order to identify the National Institute for Food and Drug Control (strain 140623) and Shin Biofermin S (strain SBS-1, one of the cocci), which are widely used clinically, the present study sequenced and analyzed these two strains. The biochemical characteristics, gas chromatography and mass spectrometry results of 140623 and SBS-1 revealed that the two strains were more similar to E. faecium than E. faecalis. The genomes of 140623 and SBS-1 contained 2,812,926 bp and 2,797,745 bp, respectively, based on Illumina HiSeq 2000 sequencing. Phylogenetic analysis demonstrated that 140623 and SBS-1 belonged to the phylogenetic group of E. faecium. The Gene Ontology, Kyoto Encyclopedia of Genes and Genomes and Clusters of Orthologous Groups classifications of the two sequenced genomes were highly conserved with reference to E. faecium strains. A total of 6 putative virulence-associated genes, 15 antibiotic resistance genes and 31 genes associated with bacterial toxins were identified from 140623 and SBS-1, representing their resistance mechanisms in natural environments and their potential for clinical use in food and drug safety.
\end{abstract}

\section{Introduction}

Enterococcus belongs to the Enterococcaceae family, the members of which are symbiotic bacteria in the human intestine (1). Enterococcus infections may occur in the urinary tract and in meningitis, diverticulitis, bacteremia and endocarditis infections with high mortality rate of $\sim 61 \%$ in Portugal (2). Enterococcus is used as a probiotic to improve the intestinal

Correspondence to: Dr Bin Zhu, Guangxi Institute for Food and Drug Control, 9 Qinghu Road, Nanning, Guangxi Zhuang Autonomous Region 530021, P.R. China

E-mail: zhubin1226@sina.com

Key words: Enterococcus faecium, genome, Hiseq2000, antibiotic resistance genes, virulence environment when treating bacterial diarrhea (3). There are two main advantages to Enterococcus that make it the most popular edible probiotic in animals and humans: i) In the gastrointestinal (GI) tract, Enterococcus competes with pathogens and thus decreases their virulence (2); ii) Enterococcus resists acid stress and cannot be digested by GI-secreted digestive juice (4). E. faecalis and E. faecium are used as digestive agents for the treatment of diarrhea caused by flatulence and indigestion (2). However, these two bacteria are difficult to distinguish morphologically. Species identification and genome characterization of these strains may elucidate therapeutic strategies for bacterial infection and probiotic treatment therapy.

The two bacteria were originally assigned to the Streptococcus genus (2). In 1984, Schleifer et al (5) indicated that they belonged to the Enterococcus genus via DNA-DNA and DNA-rRNA hybridization. These changes were also confirmed in 'Bergey's Manual of Systematic Bacteriology Volume 3: The Firmicutes' in 2009, such that 'Streptococcus faecalis' was revised to Enterococcus faecalis, and 'Streptococcus faecium' was revised to E. faecium (6). Due to previous changes in species names, the bacterial standard used by a number of manufacturers is inaccurate, which leads to incorrect or inconsistent identification of bacteria. However, numerous species of Enterococci possess the ability to transfer and carry antibiotic resistance genes (7). Therefore, the accurate definition and identification of the different sources of the strains in production is essential, as these issues may affect drug production and safe use.

Full bacteria genome sequencing provides an unprecedented method of investigating the biological processes and evolutionary characteristics of bacteria. Using these data, comparative analyses can be made to identify phylogenetic relationships among species, obtain molecular markers for stains and to investigate drug-resistant genes. To date, 527 E. faecalis and 778 E. faecium draft genomes have been submitted to GenBank (http://www.ncbi.nlm.nih.gov/genome; September 3, 2017). Although E. faecalis and E. faecium are difficult to distinguish morphologically, different genome structures have been identified between the two species. The gene order of E. faecium is also significantly different from that of $E$. faecalis according to complete genome data analysis (8). In addition, using competitive DNA hybridization, Shanks et al (9) demonstrated that a number of cell-surface proteins were different between E. faecalis and E.faecium, 
which may assist in developing biomarkers for the identification of these species.

Shin Biofermin $\mathrm{S}$ is the most widely used commercial medicine for the treatment of dyspepsia, abdominal distension and diarrhea (10). Unfortunately, the ingredients, including the species of strains, are yet to be fully elucidated. The present study isolated the strain from Shin Biofermin S. using the Illumina HiSeq 2000 platform, after which the complete genomes of two E. faecium strains were determined and annotated. The phylogenetic relationships between these two strains and their genome characteristics were identified and compared with those of other strains of E. faecalis and E. faecium. This genomic information may provide a reference genome data set of E. faecium strains to aid further investigations into the ecological and functional diversity of E. faecium. In addition, the results of the present study identified the species of the two clinically applied strains, 140623 and SBS-1, which may guide the further production of edible probiotics and the optimization of bacterial species.

\section{Materials and methods}

Isolation of strains and DNA isolation. Strain 140623 was isolated from the National Institute for Food and Drug Control as a control for the production of Lactasin Tablets, and SBS-1 was isolated from Shin Biofermin S (cat. no. X20000191, Biofermin Pharmaceutical Co., Ltd.; https://www.biofermin. co.jp/). The 140623 bacterial powder was added to MRS medium (Tiangen Biotech Co., Ltd.) and cultured for $48 \mathrm{~h}$ at $37^{\circ} \mathrm{C}$. For the isolation of SBS-1, $1 \mathrm{~g}$ Shin Biofermin S Tablet was first diluted in $9 \mathrm{ml} 7.5 \%$ sodium chloride solution and then cultured using $0.1 \mathrm{ml}$ diluted test solution for $48 \mathrm{~h}$ at $37^{\circ} \mathrm{C}$ in MRS medium. Following culture, genomic DNA was isolated using a Bacterial Genome DNA Extraction kit (Tiangen Biotech Co., Ltd.) according to the manufacturer's protocol.

Biochemical identification of 140623 and SBS-1. The 140623 and SBS-1 strains were identified using tests for the VITEK $^{\circledR} 2$ GP ID card in the VITEK 2 Compact 30 system (BioMérieux SA; https://www.biomerieux.com). The growth and biochemical characteristics of these strains were assessed by the Bacteria Preservation Center from the Institute of Microbiology, Chinese Academy of Sciences (Beijing, China). The DuPont ${ }^{\mathrm{TM}}$ RiboPrinter ${ }^{\circledR}$ System (Hygiena, LLC) was used to identify species by ribotyping (DuPont).

Analysis of fatty acid components by gas chromatography-mass spectrometry $(G C-M S)$. In this experiment, the Enterococcus faecium strain CGMCC1.131, provided by The China General Microbiological Culture Collection Center (Institute of Microbiology; Chinese Academy of Sciences) was used as control. Strains were grown in $100 \mathrm{ml}$ MRS medium at $37^{\circ} \mathrm{C}$ for $48 \mathrm{~h}$, centrifuged at $3000 \mathrm{x} \mathrm{g}$ for $10 \mathrm{~min}$ at room temperature and washed three times with deionized water. Collected bacteria were subsequently dried at $60^{\circ} \mathrm{C}$ for $3 \mathrm{~h}$. The fatty acid components were analyzed using an MS 5975C (Agilent Technologies GmbH). The gas chromatography column was an HP-5MS (30 $\mathrm{m} \times 0.25 \mathrm{~mm} \times 0.25 \mu \mathrm{m})$ (Agilent Technologies $\mathrm{GmbH}$ ). The carrier gas was helium.
Table I. Biochemical identification of two strains.

\begin{tabular}{|c|c|c|}
\hline Test items & 140623 & SBS-1 \\
\hline Gram staining & Positive & Positive \\
\hline Cell shape & spherical & spherical \\
\hline Spore & - & - \\
\hline Catalase test & - & - \\
\hline Oxidase test & - & - \\
\hline Growth in air & + & + \\
\hline Growth at $45^{\circ} \mathrm{C}$ & + & + \\
\hline Growth at $10^{\circ} \mathrm{C}$ & + & + \\
\hline $6.5 \% \mathrm{NaCl}$ growth & + & + \\
\hline pH 9.6 growth & + & + \\
\hline $\mathrm{pH} 4.5$ growth & - & - \\
\hline D-glucose & + & + \\
\hline D-fructose & + & + \\
\hline D-mannose & + & + \\
\hline D-ribose & + & + \\
\hline D-xylose & - & - \\
\hline L-xylose & - & - \\
\hline D-galactose & + & + \\
\hline D-arabinose & - & - \\
\hline L-arabinose & + & + \\
\hline L-sorbose & - & - \\
\hline L-rhamnose & - & - \\
\hline Lactose & + & + \\
\hline Sucrose & + & + \\
\hline Maltose & + & + \\
\hline Trehalose & + & + \\
\hline Melibiose & + & + \\
\hline Cellobiose & + & + \\
\hline Melezitose & - & - \\
\hline Raffinose & - & - \\
\hline Sorbitol & - & - \\
\hline Mannitol & + & + \\
\hline Sodium gluconate & + & + \\
\hline Esculin & + & + \\
\hline Salicin & + & + \\
\hline Amygdalin & + & + \\
\hline Species & $\begin{array}{c}\text { Enterococcus } \\
\text { faecium }\end{array}$ & $\begin{array}{c}\text { Enterococcus } \\
\text { faecium }\end{array}$ \\
\hline
\end{tabular}

- , negative result, + , positive result.

The warming program was $120^{\circ} \mathrm{C}$ for $5 \mathrm{~min}$, the temperature was raised to $240^{\circ} \mathrm{C}\left(6^{\circ} \mathrm{C} / \mathrm{min}\right)$ and maintained for $10 \mathrm{~min}$. The temperature was then raised to $260^{\circ} \mathrm{C}\left(10^{\circ} \mathrm{C} / \mathrm{min}\right)$ and maintained for $2 \mathrm{~min}$. The inlet temperature was $250^{\circ} \mathrm{C}$, the split ratio was $10: 1$, the flow was 1.0 and the injection volume was $1 \mu 1$. The mass spectrometry conditions were an ion source with an electron energy of $70 \mathrm{eV}$, a 3 min solvent delay, an electron multiplier voltage gain factor $(=1)$ mode with a full mass scan range of 35-450 amu and a sampling frequency of 2 . Multiple reaction monitoring transitions was used to monitor 


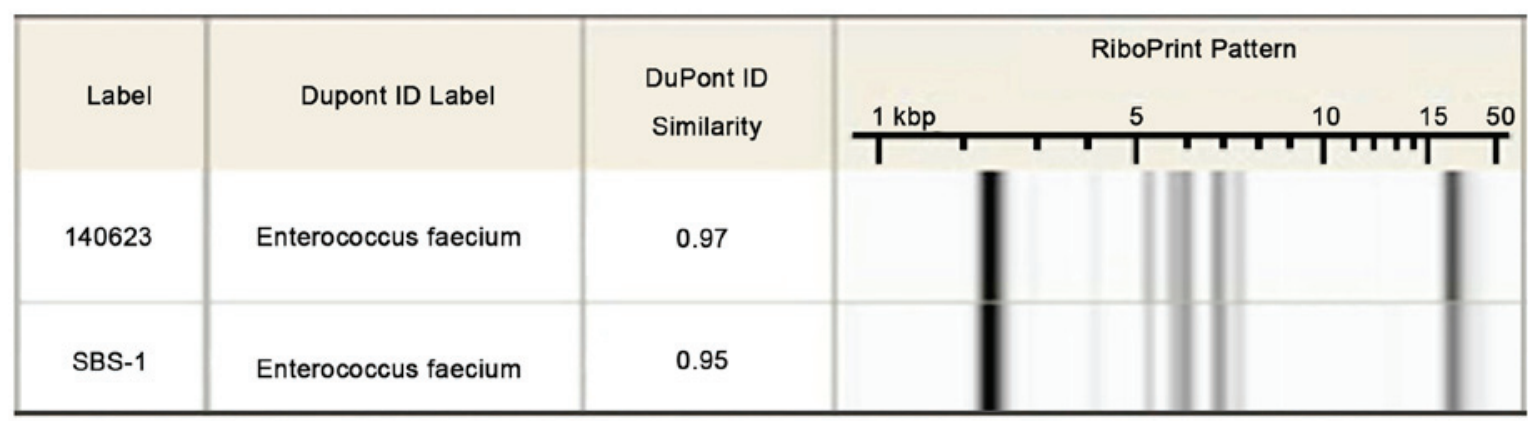

Figure 1. RiboPrinter ribotyping results of the National Institute for Food and Drug Control (140623) and Shin Biofermin S (SBS-1).

entire eluate with dwell time of $100 \mathrm{~ms}$. The optimum voltage of the first octupole was performed. To analyze the fatty acid methyl esters, $50 \mathrm{mg}$ of samples or fatty acid standards was dissolved with $2 \mathrm{ml}$ boron trifluoride $14 \%$ in methanol solution and sealed by $\mathrm{N}_{2}\left(250^{\circ} \mathrm{C}\right)$, at a flow rate of $5 \mathrm{l} / \mathrm{min}$ and a nebulizer gas pressure of $20 \mathrm{psi}$, immediately following $10 \mathrm{~min}$ of ultrasonic shock. After incubation in a $70^{\circ} \mathrm{C}$ water bath for $30 \mathrm{~min}, 2 \mathrm{ml} \mathrm{N}$-hexane was added and mixed. The supernatant was collected and washed with $1 \mathrm{ml} \mathrm{N}$-hexane following filtration with $0.22 \mu \mathrm{m}$ filter membranes and GC-MS analysis.

Genome sequencing and assembly. The genomes of 140623 and SBS-1 were sequenced using the Illumina HiSeq 2000 platform (Illumina, Inc.). Genomic DNA $(1 \mu \mathrm{g})$ from each strain was fragmented randomly and purified by electrophoresis using $1.0 \%$ agarose gel (Tiangen Biotech Co., Ltd.). The gel was then visualized by ethidium bromide staining under UV light. The DNA fragments were connected to adaptors and sequenced using the Illumina HiSeq 2000. The raw reads were used for de novo assembly by SOAPdenovo software (version 2.04; http://soap.genomics.org.cn/soap denovo.html) (11). The de novo assembly was assessed by kmer and GC depth analysis. Kmer was calculated using Meryl (https://github.com/marbl/meryl) with parameter set to kmer=15. SOAP2 reads was used to map the genome and calculate GC depth (11). E. faecium T110 strain was used as control for the comparison of sequences.

Genome annotation. The complete genome sequences of 140623 and SBS-1 were annotated using the Basic Local Alignment Search Tool (BLAST, version: 2.2.27) (12) against the Gene Ontology (GO) (13), Kyoto Encyclopedia of Genes and Genomes (KEGG) (14-16), Swiss-Prot (17), non-redundant database and Clusters of Orthologous Groups $(C O G)(18,19)$ databases $\left(\right.$ Expect $(E)$ Value $\left.=1 \times 10^{-100,000}\right)$. The colinearity analysis of genome was performed by mapping the unigenes with high sequence similarity hits (threshold value $\left.=1 \times 10^{-30}\right)(20)$. The pathogenicity and drug resistance of animal pathogen analyses were determined using type III secretion system (T3SS) prediction, which was performed using EffectiveT3 (version 1.0.1) (21), Virulence Factors Database (version 20130128) (22) and Antibiotic Resistance Genes Database (version 1.1) (23) database annotations. Repeated sequence analyses, including small satellite sequences and microsatellite sequences, were performed using Tandem Repeat Finder (24). Ribosomal RNA (rRNA) and bacterial small RNA (sRNA) were predicted by searching the Rfam databases (version 10.1) (25). The remainder of the potential noncoding RNAs was predicted using RNAmmer (version 1.2) (26) and tRNAscan (version: 1.23) (27) software. Potential virulence-associated genes were predicted by BLAST queries against the pathogen-host interaction gene database (http://www.phi-base.org). Genes of antibiotic resistance were predicted using Antibiotic Resistance Genes Database (ARDB) (https://ardb.cbcb.umd.edu/).

\section{Results}

Biochemical identification results of 140623 and SBS-1. Biochemical identification tests revealed that the two tested strains were Gram-positive, spherical bacterium. No spores were observed when culturing the bacteria. The catalase and oxidase tests were negative in both strains. These bacteria could grow in air and were able to survive and multiply at either 10 or $45^{\circ} \mathrm{C}$. D-glucose, D-fructose, D-mannose, D-ribose, D-galactose, L-arabinose, lactose, sucrose, maltose, trehalose, melibiose, cellobiose, mannitol, sodium gluconate, esculin, salicin and amygdalin tests were all positive, while the D-xylose, L-xylose, D-arabinose, L-sorbose, L-rhamnose, melezitose, raffinose and sorbitol tests exhibited negative results in the bacteria (Table I). These results indicated that the two strains were E. faecium. The RiboPrinter ribotyping results also indicated the two strains demonstrated high similarity with E.faecium (Fig. 1).

GC-MS analysis of fatty acid components. The two strains revealed a GC-MS result of C14:0, C16:1 $1^{\Delta 9}, \mathrm{C} 16: 0, \mathrm{C} 18: 1^{\Delta 9}$ and C18:0 peaks. A specific 2-C8H17-C19:0 peak was observed in the tested strains. The concentrations of C14:0, C16:1 ${ }^{\Delta 9}, \mathrm{C} 16: 0$, $\mathrm{C} 18: 1^{\Delta 9}, \mathrm{C} 18: 0$ and 2-C8H17-C19:0 were 6.60, 9.61, 17.50, 28.14, 3.93 and 36.29 , respectively, in strain 140623 . The concentrations of C14:0, C16:1 $1^{\Delta 9}, \mathrm{C} 16: 0, \mathrm{C} 18: 1^{\Delta 9}, \mathrm{C} 18: 0$, and 2-C8H17-C19:0 were $6.29,8.63,17.58,30.27,3.95$ and 32.47 , respectively, in SBS-1. The two strains had similar ratios of C14:0, C16:1 ${ }^{\Delta 9}$, C16:0, C18:1 ${ }^{\Delta 9}, \mathrm{C} 18: 0,2-\mathrm{C} 8 \mathrm{H} 17-\mathrm{C} 19: 0$ (1.0:1.5:2.7:4.3:0.6:5.5 in $140623 ; 1.0: 1.4: 2.8: 4.8: 0.6: 5.2$ in SBS-1) with the control (1.0:1.4:2.7:4.3:0.5:5.0 in control) E. faecium (Table II).

Genome assembly of 140623 and SBS-1. Illumina HiSeq 2000 generated 2,132 $\mathrm{Mb}$ and 2,413 $\mathrm{Mb}$ raw data from 140623 and 
Table II. Relative content of standard strains and the strains of lactasin tablets (\%).

\begin{tabular}{lccccccc}
\hline Strains & $\mathrm{C} 14: 0$ & $\mathrm{C} 16: 1^{\Delta 9}$ & $\mathrm{C} 16: 0$ & $\mathrm{C} 18: 1^{\Delta 9}$ & $\mathrm{C} 18: 0$ & 2-C $_{8} \mathrm{H}_{17}-\mathrm{C} 19: 0$ & Content ratio of component \\
\hline 140623 & 6.60 & 9.61 & 17.50 & 28.14 & 3.93 & 36.29 & $1.0: 1.5: 2.7: 4.3: 0.6: 5.5$ \\
SBS-1 & 6.29 & 8.63 & 17.58 & 30.27 & 3.95 & 32.47 & $1.0: 1.4: 2.8: 4.8: 0.6: 5.2$ \\
Enterococcus & 6.68 & 9.66 & 17.85 & 28.56 & 3.43 & 33.5 & $1.0: 1.4: 2.7: 4.3: 0.5: 5.0$ \\
faecium control & & & & & & & \\
\hline
\end{tabular}

Table III. Summary of genome assembly of the two strains.

\begin{tabular}{lcc} 
A, 140623 & & \\
\hline Characteristic & Scaffold & Contig \\
\hline Total number, $\mathrm{n}$ & 6 & 63 \\
Total length, bp & $2,812,926$ & $2,765,223$ \\
N50, bp & $2,713,725$ & 125,144 \\
N90, bp & $2,713,725$ & 36,082 \\
Max length, bp & $2,713,725$ & 460,559 \\
Min length, bp & 613 & 211 \\
GC content, $\%$ & 38.22 & 38.22 \\
\hline B, SBS-1 & & \\
\hline Characteristic & & Contig \\
\hline Total number, $n$ & Scaffold & 38 \\
Total length, bp & 10 & $2,754,045$ \\
N50, bp & $2,797,745$ & 208,101 \\
N90, bp & $2,177,304$ & 49,841 \\
Max length, bp & 415,863 & 447,113 \\
Min length, bp & $2,177,304$ & 232 \\
GC content, $\%$ & 1,148 & \\
\hline
\end{tabular}

SBS-1, respectively. The sequence data of which are available under NCBI BioProject number PRJNA549093. The de novo assembly of 140623 generated 6 scaffolds (N50, 2,713,725 bp) and 63 contigs (N50, 125,144 bp), indicating that the 140623 genome had $2,812,926$ bp nucleotides with $38.22 \%$ GC content (Table III). The complete genome of SBS-1 contained 2,797,745 bp nucleotides with $38.25 \%$ GC content, which had 10 scaffolds (N50, 2,177,304 bp) and 38 contigs (N50, 208,101 bp; Table IV).

The genome characteristics, including gene distribution and genome GC content distribution. A higher GC skew (-) value was found in SBS-1, which may due to genome variation between the two strains (Fig. 2A and B). In the present study, the T110 strain was used as a control to compare the genome sequences between the strains. The collinearity analysis of the two strains and T110 demonstrated that 140623 and SBS-1 were most closely associated with sequences from T110 (Fig. 2C and D). Similar arrangement of sequences from the 140623 and SBS-1 genomes was found by comparing them with sequences from the T110 genome. The genome collinearity
Table IV. Genome component of the two strains.

\begin{tabular}{lcc}
\hline & 140623 & SBS-1 \\
\hline Genome size, bp & $2,812,926$ & $2,797,745$ \\
GC content, \% & 38.23 & 38.26 \\
Gene number & 2,766 & 2,734 \\
Gene length, bp & $2,457,726$ & $2,441,361$ \\
Gene average length, bp & 889 & 893 \\
Gene length/genome, \% & 87.37 & 87.26 \\
GC content in gene region, \% & 39.07 & 39.09 \\
Intergenic region length, bp & 355,200 & 356,384 \\
GC content in intergenic region, $\%$ & 32.42 & 32.57 \\
Intergenic region length/genome, $\%$ & 12.63 & 12.74 \\
Tandem repeat number & 70 & 70 \\
Tandem repeat length, bp & 7,954 & 9,079 \\
Tandem repeat size, bp & $5-676$ & $5-692$ \\
Tandem repeat length/genome, $\%$ & 0.2828 & 0.3245 \\
Minisatellite DNA Number & 39 & 38 \\
Microsatellite DNA number & 2 & 1 \\
rRNA number & 0 & 3 \\
tRNA number & 41 & 54 \\
sRNA number & 4 & 4 \\
Genomic island number & 0 & 0 \\
Prophage number & 0 & 0 \\
\end{tabular}

rRNA, ribosomal RNA; tRNA, transfer RNA; sRNA, bacterial small RNA.

analysis of amino acid and nucleotide sequences exhibited similar arrangements in 140623 and SBS-1 compared with T110 (Fig. 2C and D).

Annotation of the 140623 and SBS-1 genomes. Gene annotations identified 2,766 genes (average gene length, $889 \mathrm{bp}$ ) and 2,734 genes (average gene length, 893 bp) from 140623 and SBS-1, respectively. Compared with the intergenic region length (140623, 355,200 bp; SBS-1, 356,384 bp), larger gene regions were identified in the 140623 genome $(2,457,726 \mathrm{bp})$ and SBS-1 genome (2,441,361 bp), representing 87.37 and $87.26 \%$ of the complete genome sequences, respectively. Higher GC contents in gene regions (140623, 39.07\%; SBS-1, 39.09\%) were observed when compared with intergenic regions (140623, $32.42 \%$; SBS-1, 32.57\%). Both genomes contained 70 tandem repeat sequences (7,954 bp in 140623, 9,079 bp in SBS-1), which accounted for 0.2828 and $0.3245 \%$ of the genomes of 140623 

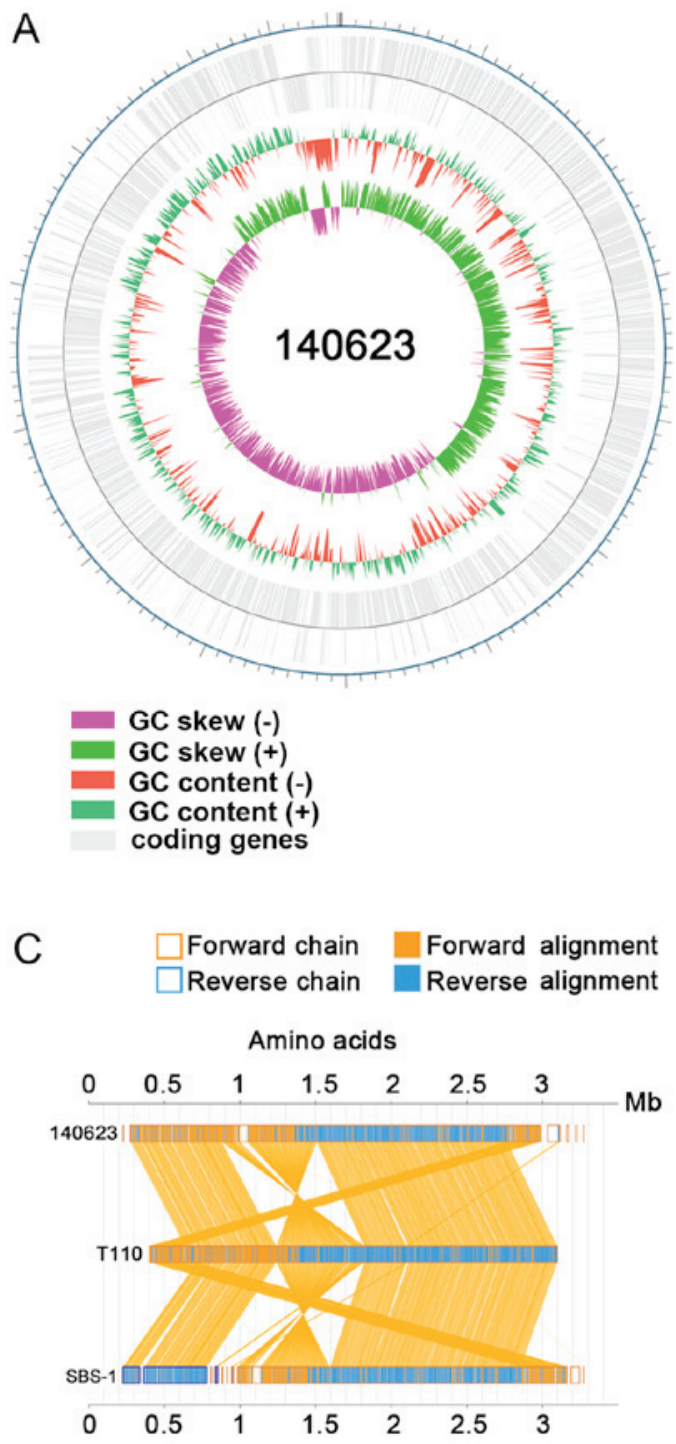

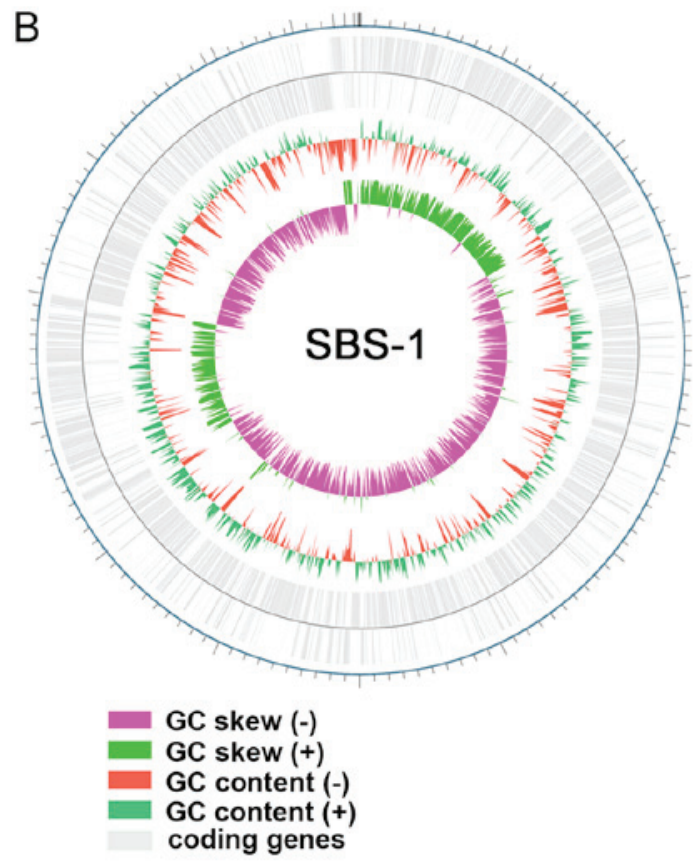

$\square$ Forward chain $\quad$ Forward alignment
$\square$ Reverse chain

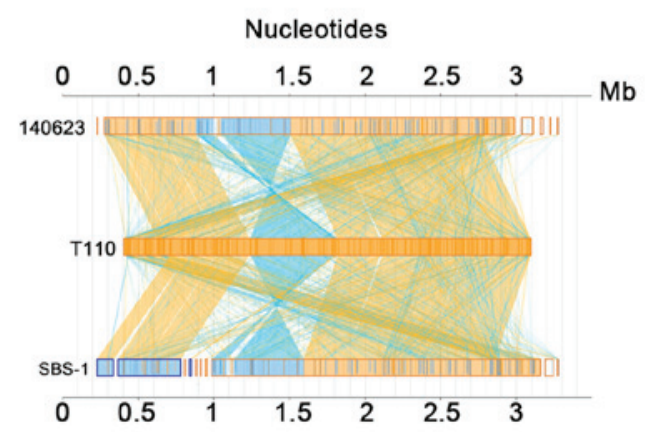

Figure 2. Genome features and comparative analysis of National Institute for Food and Drug Control (140623) and Shin Biofermin S (SBS-1). Genome features of (A) 140623 and (B) SBS-1. (C) Collinearity analysis of amino acid sequences of protein and (D) nucleotide sequences between the 140623 , T110 and SBS-1 strains.

and SBS-1, respectively. In 140623, 39 minisatellite DNA sequences, 2 microsatellite DNA sequences, 41 transfer RNAs (tRNAs) and 4 sRNAs were identified. The SBS-1 genome contained 38 minisatellite DNA sequences, 1 microsatellite DNA sequence, 3 rRNAs, 54 tRNAs and 4 sRNAs (Table IV).

The distribution of GO categories was similar between the 140623 and SBS-1 genomes. In 140623 and SBS-1, the genes associated with 'catalytic activity', 'metabolic process', 'cellular process' and 'binding' were abundant (Fig. 3). The core genes were primarily involved in the following KEGG categories: 'Environmental Information Processing-Membrane Transport' (478 genes in 140623 and 474 genes in SBS-1) and 'Metabolism-Carbohydrate Metabolism' (360 genes in 140623 and 350 genes in SBS-1; Fig. 4). According to the COG classification, 1,745 genes in 140623 and 1,730 genes in SBS-1 were included. In the categories other than 'Carbohydrate transport and metabolism', 'Translation, ribosomal structure and biogenesis', 'Transcription' and 'Replication, recombination and repair', the two strains had the same gene numbers (Fig. 5).
Virulence- and drug resistance-associated genes. A total of 6 putative virulence-associated genes were identified from 140623 and SBS-1 genomes, including AphA, CBL1, CPA1, GyrA, MGG_00383 and SOD2 (Table SI). Analysis using ARDB revealed that there were only a few potential genes of antibiotic resistance (15 genes in both 140623 and SBS-1 genomes; Table SII). The complete sequences of the 140623 and SBS-1 genomes each exhibited 31 genes associated with bacterial toxins according to vfdb annotation (Table SIII).

\section{Discussion}

As a popular medicine for probiotic therapy to treat with patients with diarrhea, the safety of Shin Biofermin S is paramount. Therefore, in the present study, the species of the SBS-1 strain isolated from a commercial medicine (Shin Biofermin S) was determined via genome sequencing. Species identification is important for evaluating safety and is favorable for formulating therapeutic strategies. The scientific names of E.faecium 

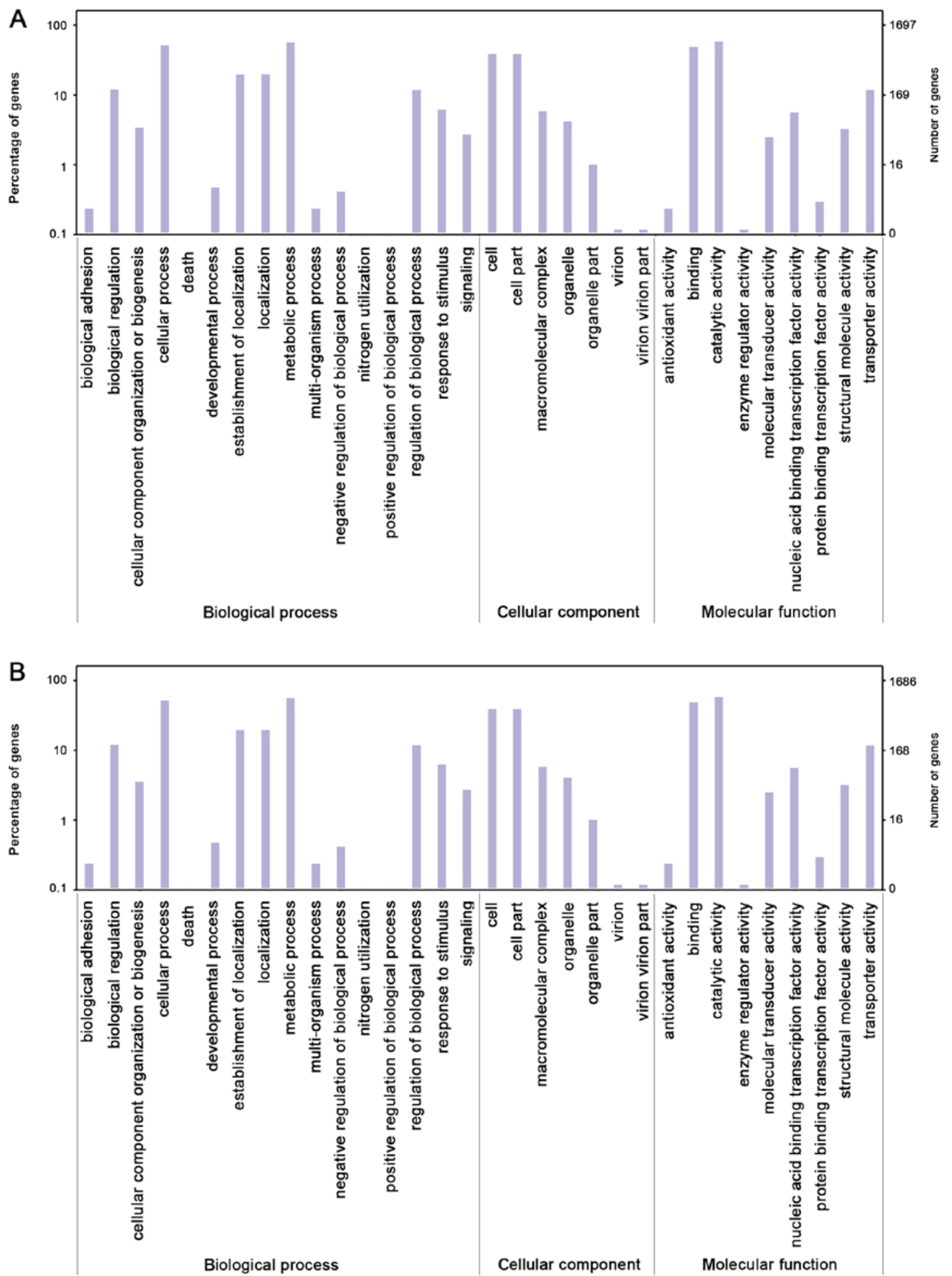

Figure 3. Gene Ontology annotations of the (A) National Institute for Food and Drug Control (140623) and (B) Shin Biofermin S (SBS-1) genes.

have been changed several times. Streptococcus faecalis and Streptococcus faecium were separate until 1984, when DNA-DNA and DNA-rRNA hybridization tests revealed that they belonged to Enterococcus instead (5). Bergey's Manual of Systematic Bacteriology Volume 3: The Firmicutes also demonstrated this result (6), such that Streptococcus faecalis was renamed Enterococcus faecalis. In the food and health industry, E. faecium and E. faecalis have been used to improve the intestinal environment by regulating its inflammatory status and the gut microflora (2). However, the definition of strains is inaccurate among different companies. The bacterial strain is the only active ingredient in probiotics. Thus, if the identity of the original strain species is not assured, its safety cannot be guaranteed. The present study identified two E. faecium strains 
A

KEGG pathway classification
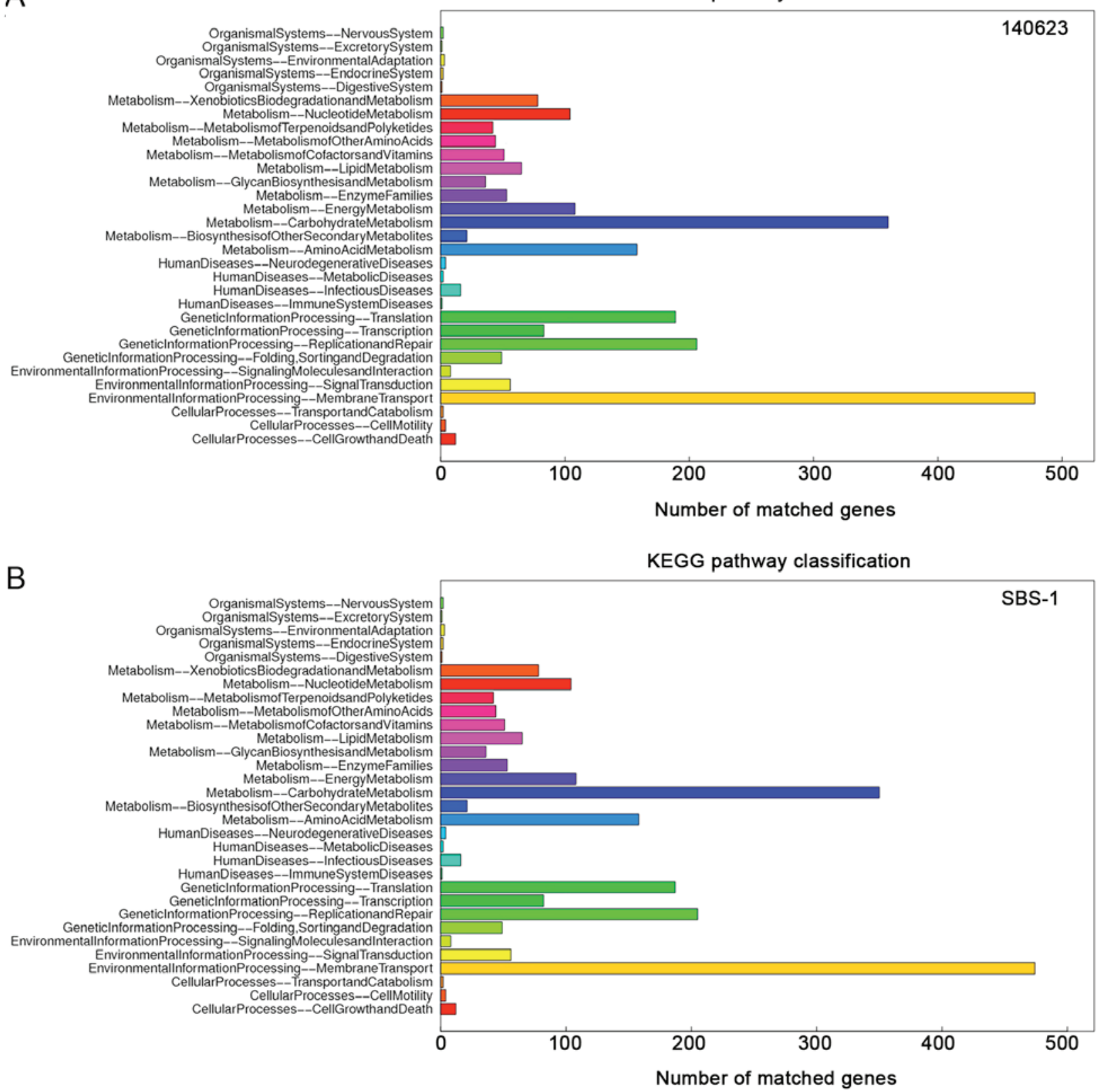

Figure 4. Kyoto Encyclopedia of Genes and Genomes annotation of (A) National Institute for Food and Drug Control (140623) and (B) Shin Biofermin S (SBS-1) genes.

from the National Institute for Food and Drug Control, and Shin Biofermin S. Biochemical identification, gene fingerprinting, fatty acid component analysis and genome sequencing were all used to clarify the species of these two strains.

Growth and sugar component tests classified the two strains into E. faecium. The growth of E. faecium requires high-quality culture medium, such as agar with human, rabbit or horse blood. This species produced L-arabinose, in line with a previous report (28), while E. faecium could not generate L-arabinose. In addition, the RiboPrinter automatic microbial genetic fingerprint identification system, which is a tool designed for the identification of bacteria, suggested that the two strains were E. faecium. A number of studies have demonstrated that this method is a highly targeted and effective approach to identify different species. Previously, the two strains seemed to be E.faecalis $(2,5)$, while the present analyses strongly supports E. faecium.

The fatty acid composition of the strains was analyzed in the present study via GC-MS, which also supported the classification of the two strains as E. faecium. The characteristic peaks of C14:0, C16:1 $1^{\Delta 9}, \mathrm{C} 16: 0, \mathrm{C} 18: 1^{\Delta 9}, \mathrm{C} 18: 0$ and 2-C8H17-C19:0 were similar between the two strains. This result demonstrated the high homology between 140623 and SBS-1. The fatty acid component of these two strains was also similar to previously reported E. faecium (29).

The genome sizes of the two strains were 2,812,926(140623) and 2,797,745 (SBS-1) bp. Zhong et al (30) reported five strains of E. faecium genome sizes ranging from 2.64-2.99 Mb. The genome sizes identified in the present study were also in this range. Another report indicated that the genome of an 
A COG function classification

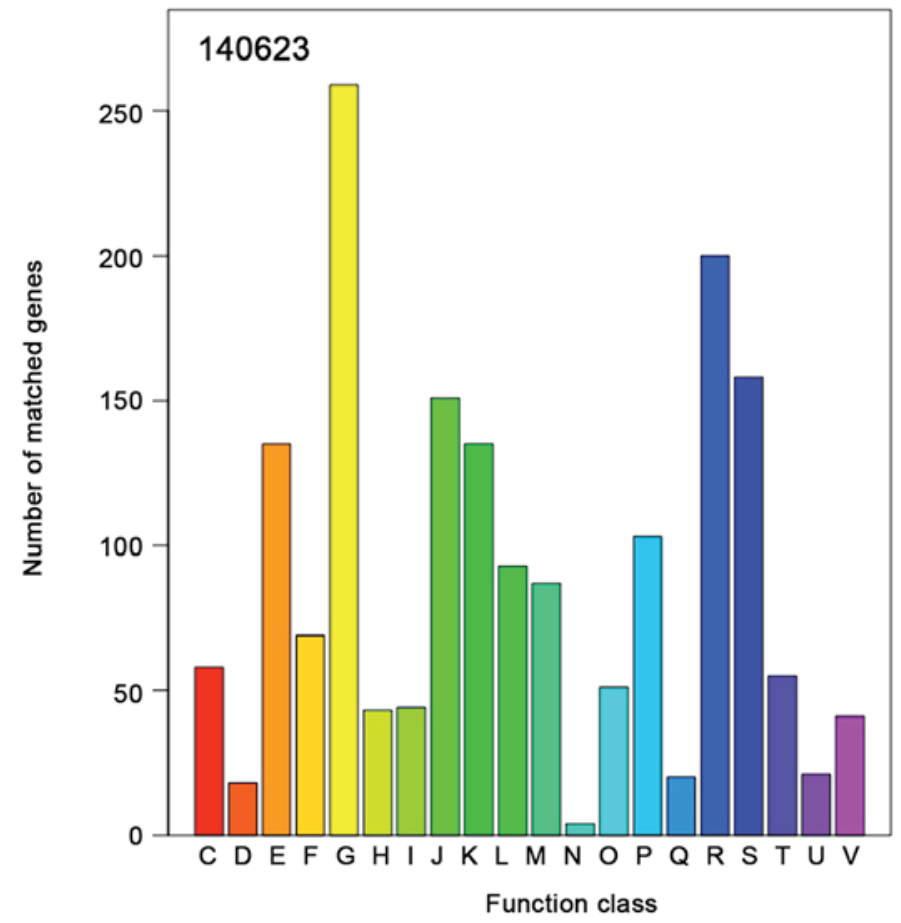

C: Energy production and conversion

D: Cell cycle control, cell division, chromosome partitioning

E: Amino acid transport and metabolism

F: Nucleotide transport and metabolism

G: Carbohydrate transport and metabolism

$\mathrm{H}$ : Coenzyme transport and metabolism

I: Lipid transport and metabolism

$\mathrm{J}$ : Translation, ribosomal structure and biogenesis

K: Transcription

L: Replication, recombination and repair

M: Cell wall/membrane/envelope biogenesis

$\mathrm{N}$ : Cell motility

O: Posttranslational modification, protein turnover, chaperones

$P$ : Inorganic ion transport and metabolism

Q: Secondary metabolites biosynthesis, transport and catabolism

$\mathrm{R}$ : General function prediction only

S: Function unknown

$\mathrm{T}$ : Signal transduction mechanisms

$\mathrm{U}$ : Intracellular trafficking, secretion, and vesicular transport

V: Defense mechanisms

B

B COG function classification

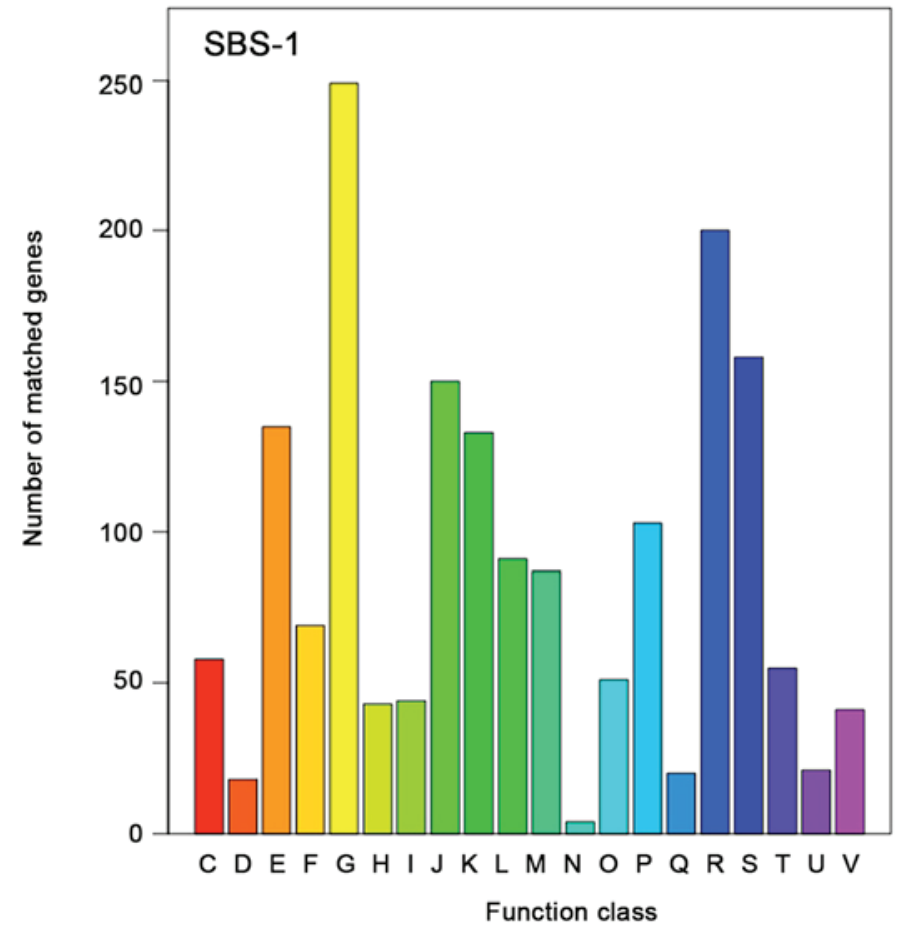

C: Energy production and conversion

D: Cell cycle control, cell division, chromosome partitioning

E: Amino acid transport and metabolism

F: Nucleotide transport and metabolism

G: Carbohydrate transport and metabolism

$\mathrm{H}$ : Coenzyme transport and metabolism

I: Lipid transport and metabolism

$\mathrm{J}$ : Translation, ribosomal structure and biogenesis

K: Transcription

L: Replication, recombination and repair

$\mathrm{M}$ : Cell wall/membrane/envelope biogenesis

$\mathrm{N}$ : Cell motility

O: Posttranslational modification, protein turnover, chaperones

$P$ : Inorganic ion transport and metabolism

Q: Secondary metabolites biosynthesis, transport and catabolism

$\mathrm{R}$ : General function prediction only

S: Function unknown

T: Signal transduction mechanisms

$\mathrm{U}$ : Intracellular trafficking, secretion, and vesicular transport

V: Defense mechanisms

Figure 5. Clusters of Orthologous Groups annotation of (A) National Institute for Food and Drug Control (140623) and (B) Shin Biofermin S (SBS-1) genes.

E. faecium strain isolated from the bloodstream of a patient in Melbourne, Australia, contained 3 circular plasmids and a $2.9 \mathrm{Mb}$ chromosome (31). The chromosome of E. faecium TX16 was 2,698,137 bp (8). In general, it appears that genome size changes among different strains throughout evolution.

The genomes of 140623 and SBS-1 had 2,766 and 2,734 protein-coding open reading frames (ORFs), respectively.
When three $E$. faecium were isolated from bovine feces, it was revealed that they contained 2,719, 2,665 and 2,659 genes, respectively (32). In E. faecium TX16, 2,703 protein-coding ORFs were identified on the chromosome and the three plasmids contained 43, 85 and 283 ORFs, respectively (8). These previous reports indicate that the genomes of E. faecium strains from different sources have similar gene counts, indi- 
cating evolutionarily conserved and similar functions. Similar gene annotations, such as GO, KEGG and COG analyses, also supported this conclusion.

Virulence- and drug resistance-associated genes in $E$. faecium were limited compared with the results of E. faecalis. The present results demonstrated that AphA, CBL1, CPA1, GyrA, MGG_00383 and SOD2 were exhibited by the two strains and may be associated with pathogen-host interaction. CBL1 of Fusarium graminearum has been reported to be associated with pathogenicity, indicating a role in host infection (33). Waters et al (34) demonstrated the ciprofloxacin-resistant function of GyrA. The SOD2 gene is associated with the loss of pathogenicity in bacteria (35). The present study indicated that the evolution of this species conferred resistance mechanisms in natural environments and industrial environments. These resistance mechanisms provide potential for clinical use in food and drug safety.

The results of the present study indicated that the species of 140623 and SBS-1 was E. faecium. The biochemical assays and RiboPrinter ribotyping were comparable, both characterizing the strains as E. faecium; GC-MS analysis indicated specific peaks of fatty acid components that were similar to other $E$. faecium strains; genome features of 140623 and SBS-1 demonstrated high conservation of these strains as E. faecium, which was also confirmed by phylogenetic analysis.

Taken together, the results of the present study demonstrated that 140623 and SBS-1 from the National Institute for Food and Drug Control and Shin Biofermin S were E.faecium rather than $E$. faecalis. These data first elucidated the genome information of SBS-1 strain from Shin Biofermin S, which is a commercial medicine used to treat diarrhea (2). The present study demonstrated that the strain is similar with 140623 and that the variations in the SBS-1 strain served potential roles in resistance. The virulence- and drug resistance-associated genes identified in the present study, which participate in antibiotic resistance, should be further investigated. These could serve as key molecular factors for SBS-1 strain security during treatment. In conclusion, the results of the present study may therefore be valuable for distinguishing different species of the Enterococcus genus and guiding edible probiotic usage.

\section{Acknowledgements}

Not applicable.

\section{Funding}

No funding was received.

\section{Availability of data and materials}

The datasets used and/or analyzed during the present study are available from the corresponding author on reasonable request.

\section{Authors' contributions}

BZ designed the study. YQiG performed the experiments and analyzed the data. YQiG and BZ wrote the article. TZ, YQiangG and $\mathrm{ZZ}$ participated in the data processing and revision of the manuscriptt

\section{Ethics approval and consent to participate}

Not applicable.

\section{Patient consent for publication}

Not applicable.

\section{Competing interests}

The authors declare that they have no competing interests.

\section{References}

1. Rajilić-Stojanović M and de Vos WM: The first 1000 cultured species of the human gastrointestinal microbiota. FEMS Microbiol Rev 38: 996-1047, 2014.

2. Fisher K and Phillips C: The ecology, epidemiology and virulence of Enterococcus. Microbiology 155: 1749-1757, 2009.

3. Giannenas I, Papadopoulos E, Tsalie E, Triantafillou E, Henikl S, Teichmann K and Tontis D: A microbiological investigation on probiotic preparations used for animal feeding. Microbi Res 151: 167-175, 1996.

4. Klein G: Taxonomy, ecology and antibiotic resistance of Enterococci from food and the gastro-intestinal tract. Int J Food Microbiol 88: 123-131, 2003.

5. Schleifer KH and Kilpper-Bälz R: Transfer of Streptococcus faecalis and Streptococcus faecium to the Genus Enterococcus nom. rev. as Enterococcus faecalis comb. nov. and E. faecium comb. nov. Int J Syst Bacteriol 34: 31-34, 1984.

6. Vos P, Garrity G, Jones D, Krieg NR, Ludwig W, Rainey FA, Schleifer KH and Whitman W: Bergey's Manual of Systematic Bacteriology: Volume 3: The Firmicutes. Vol. 3. Springer Science \& Business Media, 2011.

7. Salyers AA, Gupta A and Wang Y: Human intestinal bacteria as reservoirs for antibiotic resistance genes. Trends Microbiol 12 . 412-416, 2004.

8. Qin X, Galloway-Peña JR, Sillanpaa J, Roh JH, Nallapareddy SR, Chowdhury S, Bourgogne A, Choudhury T, Muzny DM, Buhay CJ, et al: Complete genome sequence of Enterococcus faecium strain TX16 and comparative genomic analysis of Enterococcus faecium genomes. BMC Microbiol 12: 135, 2012.

9. Shanks OC, Santo Domingo JW and Graham JE: Use of competitive DNA hybridization to identify differences in the genomes of bacteria. J Microbiol Methods 66: 321-330, 2006.

10. Aiba Y, Ishikawa H, Shimizu K, Noda S, Kitada Y, Sasaki M and Koga Y: Role of internalization in the pathogenicity of Shiga Toxin-producing Escherichia coli infection in a gnotobiotic murine model. Microbiol Immunol 46: 723-731, 2002.

11. Luo R, Liu B, Xie Y, Li Z, Huang W, Yuan J, He G, Chen Y, Pan Q, Liu Y, et al: SOAPdenovo2: An empirically improved memory-efficient short-read de novo assembler. Gigascience 1: $18,2012$.

12. Altschul SF, Madden TL, Schäffer AA, Zhang J, Zhang Z, Miller W and Lipman DJ: Gapped BLAST and PSI-BLAST: A new generation of protein database search programs. Nucleic Acids Res 25: 3389-3402, 1997.

13. Ashburner M, Ball CA, Blake JA, Botstein D, Butler H, Cherry JM, Davis AP, Dolinski K, Dwight SS, Eppig JT, et al: Gene ontology: Tool for the unification of biology. The Gene Ontology Consortium. Nat Genet 25: 25-29, 2000.

14. Kanehisa M, Goto S, Kawashima S, Okuno Y and Hattori M: The KEGG resource for deciphering the genome. Nucleic Acids Res 32: D277-D280, 2004.

15. Kanehisa M: A database for post-genome analysis. Trends Genet 13: 375-376, 1997.

16. Kanehisa M, Goto S, Hattori M, Aoki-Kinoshita KF, Itoh M, Kawashima S, Katayama T, Araki M and Hirakawa M: From genomics to chemical genomics: New developments in KEGG. Nucleic Acids Res (Database issue) 34: D354-D357, 2006.

17. Magrane M and Consortium U: UniProt Knowledgebase: A hub of integrated protein data. Database (Oxford) 2011: bar009, 2011

18. Tatusov RL, Koonin EV and Lipman DJ: A genomic perspective on protein families. Science 278: 631-637, 1997. 
19. Tatusov RL, Fedorova ND, Jackson JD, Jacobs AR, Kiryutin B, Koonin EV, Krylov DM, Mazumder R, Mekhedov SL, Nikolskaya AN, et al: The COG database: An updated version includes eukaryotes. BMC Bioinformatics 4: 41, 2003.

20. Kurtz S, Phillippy A, Delcher AL, Smoot M, Shumway M, Antonescu C and Salzberg SL: Versatile and open software for comparing large genomes. Genome Biol 5: R12, 2004.

21. Jehl MA, Arnold R and Rattei T: Effective-a database of predicted secreted bacterial proteins. Nucleic Acids Res (Database issue) 39: D591-D595, 2011.

22. Chen L, Xiong Z, Sun L, Yang J and Jin Q: VFDB 2012 update: Toward the genetic diversity and molecular evolution of bacterial virulence factors. Nucleic Acids Res 40: D641-D645, 2012.

23. Liu B and Pop M: ARDB-Antibiotic resistance genes database. Nucleic Acids Res 37: D443-D447, 2009.

24. Benson G: Tandem repeats finder: A program to analyze DNA sequences. Nucleic Acids Res 27: 573-580, 1999.

25. Gardner PP, Daub J, Tate JG, Nawrocki EP, Kolbe DL, Lindgreen S, Wilkinson AC, Finn RD, Griffiths-Jones S, Eddy SR and Bateman A: Rfam: Updates to the RNA families database. Nucleic Acids Res 37: D136-D140, 2009.

26. Lagesen K, Hallin P, Rødland EA, Staerfeldt HH, Rognes T and Ussery DW: RNAmmer: Consistent and rapid annotation of ribosomal RNA genes. Nucleic Acids Res 35: 3100-3108, 2007.

27. Lowe TM and Eddy SR: tRNAscan-SE: A program for improved detection of transfer RNA genes in genomic sequence. Nucleic Acids Res 25: 955-964, 1997.

28. Manero A and Blanch AR: Identification of Enterococcus spp. with a biochemical key. Appl Environ Microbiol 65: 4425-4430, 1999.
29. Sakayori Y, Muramatsu M, Hanada S, Kamagata Y, Kawamoto S and Shima J: Characterization of E. faecium mutants resistant to mundticin KS, a class IIa bacteriocin. Microbiology 149: 2901-2908, 2003.

30. Zhong Z, Zhang W, Song Y, Liu W, Xu H, Xi X, Menghe B, Zhang $\mathrm{H}$ and Sun $\mathrm{Z}$ : Comparative genomic analysis of the genus Enterococcus. Microbiol Res 196: 95-105, 2017.

31. Lam MM, Seemann T, Bulach DM, Gladman SL, Chen H, Haring V, Moore RJ, Ballard S, Grayson ML, Johnson PD, et al: Comparative analysis of the first complete E. faecium genome. J Bacteriol 194: 2334-2341, 2012.

32. Beukers AG,Zaheer R, Goji N, Amoako KK, Chaves AV, Ward MP and McAllister TA: Comparative genomics of Enterococcus spp. isolated from bovine feces. BMC Microbiol 17: 52, 2017.

33. Xie QN, Jia LJ, Wang YZ, Song RT and Tang WH: High-resolution gene profiling of infection process indicates serine metabolism adaptation of Fusarium graminearum in host. Sci Bull 62: 758-760, 2017.

34. Waters B and Davies J: Amino acid variation in the GyrA subunit of bacteria potentially associated with natural resistance to fluoroquinolone antibiotics. Antimicrob Agents Chemother 41: 2766-2769, 1997.

35. Dimitrijevic L, Puppo A and Rigaud J: Superoxide dismutase activities in Rhizobium phaseoli bacteria and bacteroids. Arch Microbiol 139: 174-178, 1984.

(c) (i) () $($ This work is licensed under a Creative Commons

C. Attribution-NonCommercial-NoDerivatives 4.0 International (CC BY-NC-ND 4.0) License. 